\title{
Resistance minimum and electrical conduction mechanism in polycrystalline CoFeB thin films
}

\author{
G. Venkat Swamy ${ }^{1,2}$, P. K. Rout ${ }^{2, *}$, Manju Singh ${ }^{2}$, and R. K. Rakshit ${ }^{1,2}$ \\ ${ }^{1}$ Academy of Scientific and Innovative Research, CSIR-National Physical Laboratory Campus, \\ Dr. K. S. Krishnan Road, New Delhi - 110012, India \\ ${ }^{2}$ CSIR-National Physical Laboratory, Dr. K. S. Krishnan Road, New Delhi - 110012, Indid
}

\begin{abstract}
The temperature dependent resistance $R(T)$ of polycrystalline ferromagnetic CoFeB thin films of varying thickness are analyzed considering various electrical scattering processes. We observe a resistance minimum in $R(T)$ curves below $\simeq 29 \mathrm{~K}$, which can be explained as an effect of intergranular Coulomb interaction in a granular system. The structural and Coulomb interaction related scattering processes contribute more as the film thickness decreases implying the role of disorder and granularity. Although the magnetic contribution to the resistance is the weakest compared to these two, it is the only thickness independent process. On the contrary, the negative coefficient of resistance can be explained by electron interaction effect in disordered amorphous films.
\end{abstract}

Keywords: Ferromagnetic alloy, thin film, spintronics material

The ferromagnetic $\mathrm{CoFeB}$ has been extensively employed in various spintronics devices like magnetic tunnel junctions [1 $\left[\begin{array}{l}3 \\ ]\end{array}\right.$, spin valves [4], and spin-torque devices [5, 6] over the last decade. The electrical conduction in these devices utilising the spin degree of freedom depends on the composition, spin polarization, and crystallinity of the $\mathrm{CoFeB}$ layers. Therefore, a basic understanding of the electrical and magneto-transport properties of $\mathrm{CoFeB}$ thin films prepared under different conditions is essential for further improvement of any device based on this ferromagnetic alloy. Still there are only few reports on the electrical transport properties of $\mathrm{CoFeB}$ thin films including composites and nanotubes [7-13]. The electrical resistivity $\rho(T)$ of a crystalline film is significantly lower as compared to that of an amorphous film [11, 12]. While the crystalline films show a metallic behaviour, negative temperature coefficient of resistance has been reported for the amorphous or composite films [8, 12]. Thus, the crystalline quality plays an important role in the process of electrical conduction of these films. Moreover, the thickness of these films also controls their electrical properties as the size effects become dominant at lower thicknesses. For example, Jen et al. have reported increase of resistivity in the amorphous $\mathrm{CoFeB}$ film on lowering of the thickness [9]. However, a thorough analysis of electrical transport in crystalline $\mathrm{CoFeB}$ films is lacking.

An interesting feature of $\mathrm{CoFeB}$ systems is the presence of resistance upturn at lower temperatures 7, , 12 14]. However, there is no clear consensus on its origin due to the differences in experimental results and theoretical interpretations. Fujimori et al. have reported a $e^{-\sqrt{T}}$ dependent resistivity in the upturn region [7], which is expected for a variable range hopping conductivity in Coulomb gap [15]. A logarithmic $T$ dependent resistivity has been observed in crystalline $\mathrm{CoFeB}$ film 12] and nanotube [13] as well as amorphous ribbons 14], which can originate due to one or more of these three effects; Kondo scattering 16 or Coulomb effect in granular materials [17] or tunneling model [18]. The resis- tance minimum in variety of amorphous ferromagnetic alloys similar to $\mathrm{CoFeB}$ has been attributed to Kondo type $\ln T$ resistance [19 22 and, in some alloys, an additional minimum due to $T^{1 / 2}$ resistance has been observed [23]. Moreover, in a strong ferromagnetic material like $\mathrm{CoFeB}$, the role of magnon in electronic transport is quite important apart from other scattering mechanisms involving electron, phonon, lattice potentials due to structure etc. For example, a finite magnetic contribution to the resistivity due to electron-magnon scattering along with the structural contribution has been observed in amorphous ferromagnetic alloys like $\mathrm{FeBC}$ and FeBGe 24, 25]. All these mechanisms mostly result in various power law temperature dependence of resistivity. In order to shed some light on these aspects, we have analyzed the temperature dependent electrical transport in polycrystalline $\mathrm{Co}_{40} \mathrm{Fe}_{40} \mathrm{~B}_{20}$ thin films of varying thickness in detail. We have mainly focussed on the origin of resistance minimum observed below $\simeq 29 \mathrm{~K}$ in the light of various scattering mechanisms involved in the electrical conduction.

The thin films of $\mathrm{Co}_{40} \mathrm{Fe}_{40} \mathrm{~B}_{20}$ with nominal thicknesses ranging from 5 to $40 \mathrm{~nm}$ have been deposited on $\mathrm{SiO}_{2}(300 \mathrm{~nm}) / \mathrm{Si}(100)$ substrates using $\mathrm{KrF}$ excimer $(\lambda$ $\approx 248 \mathrm{~nm}$ ) based pulsed laser deposition technique. The growth of these films has been performed at room temperature under argon pressure of $2 \times 10^{-3}$ mbar with a typical growth rate of $0.04 \mathrm{~nm} / \mathrm{s}$. To enhance the crystallinity, the films have been subsequently annealed at $400^{\circ} \mathrm{C}$ for 1 hour under high vacuum. The details of film growth have been reported previously [12]. Figure 1 shows the grazing angle $\mathrm{X}$-ray diffraction scans for various $\mathrm{CoFeB}$ films. We have determined the actual thickness of the films from the fits to low angle scans or X-ray reflectivity (XRR) as shown in Fig. 1(b). The extracted thickness $(t)$ of the films are $6,9,20$, and $36 \mathrm{~nm}$. The high angle scan profiles show the polycrystalline nature of the films [See Fig. 1(a)]. As the film thickness increases, the intensity of (110) CoFe peak increases and it becomes much sharper as shown in Fig. 1(c), indicating 

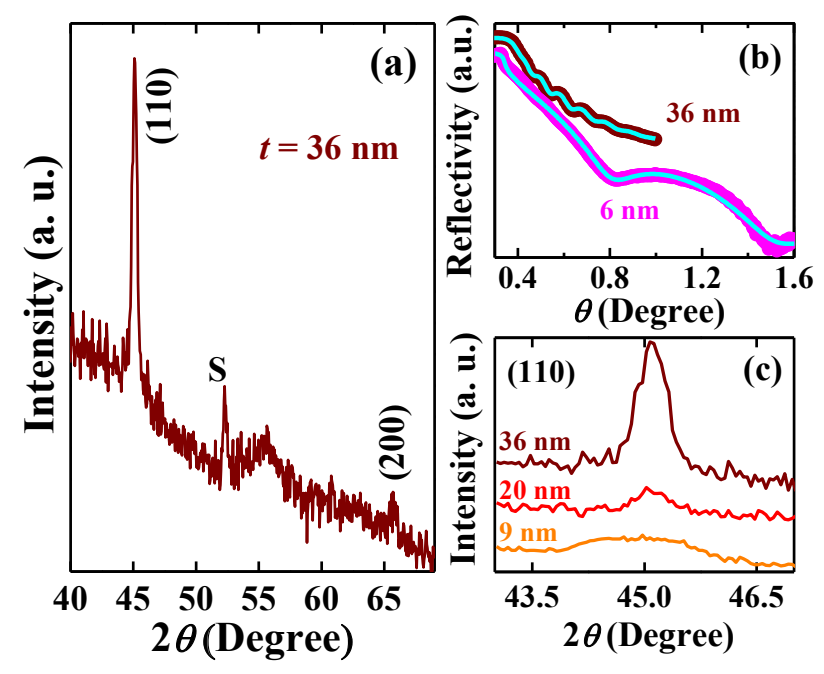

FIG. 1. (Color online) (a) X-ray diffraction pattern of $36 \mathrm{~nm}$ thick CoFeB film. The substrate peak is denoted by "S". (b) X-ray reflectivity scans for two films with $t=6$ and $36 \mathrm{~nm}$ along with the fits (solid lines). (c) X-ray diffraction pattern around (110) CoFe peak for 9, 20, and $36 \mathrm{~nm}$ thick films.

(a)

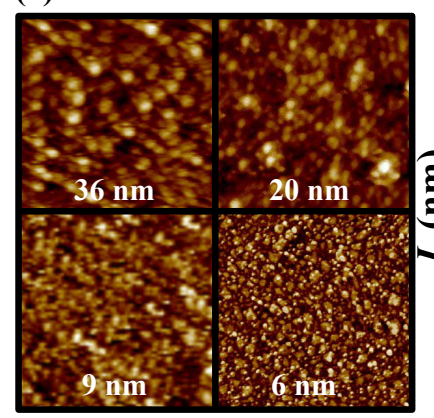

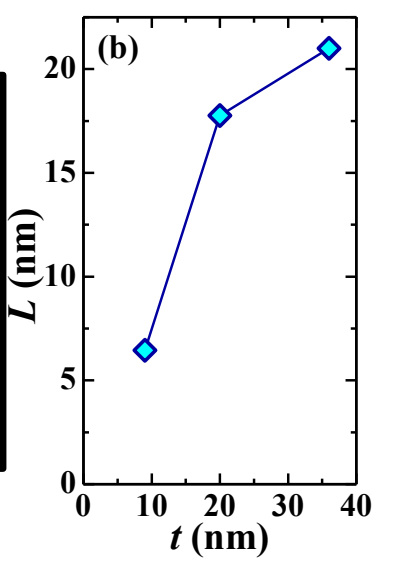

FIG. 2. (Color online) (a) The AFM images $\left(1 \times 1 \mu \mathrm{m}^{2}\right.$ area) for all films. (b) The grain size $(L)$ as a function of thickness.

better crystalline quality and the presence of larger grains in thicker films. While the fits to XRR data provide an estimation of interface roughness values as $\leq 1 \mathrm{~nm}$, the surface roughness obtained from atomic force microscopy $(\mathrm{AFM})$ scans is $\approx 1.5 \mathrm{~nm}$. Moreover, the AFM images reveal the granular nature of these films as shown in Fig. 2(a). The grain size decreases with decreasing film thickness. Similar trend can be observed from the average grain size $(L)$ determined by the Scherrer formula [See Fig. 2(b)]. We have previously reported the presence of grains with similar sizes as observed by transmission electron microscopy imaging of $\mathrm{CoFeB}$ films 12]. This variation of grain size with film thickness has significant effects on magnetic and transport properties as we will discuss now.

Figure 3(a-d) display the room temperature magnetic
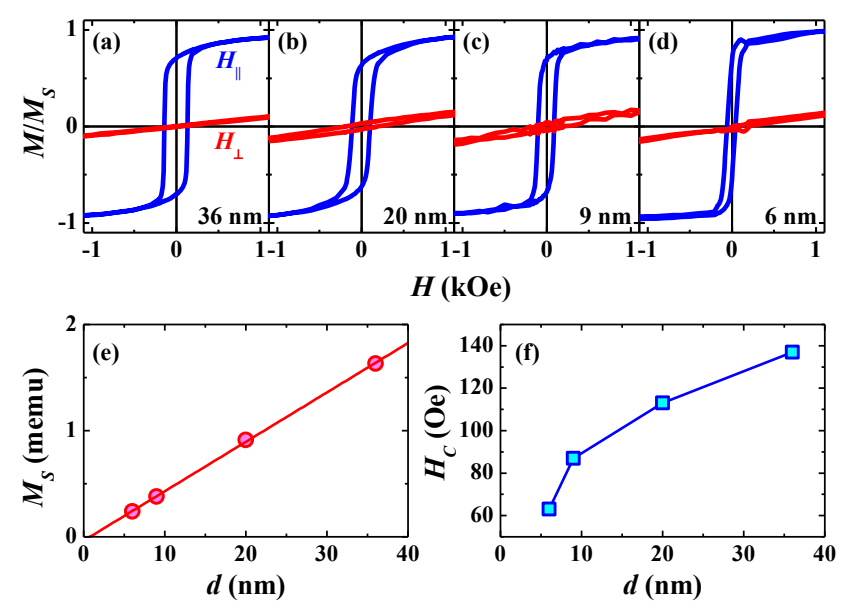

FIG. 3. (Color online) (a-d) The in-plane and out-of-plane magnetic hysteresis loops for the films with $t=6,9,20$, and $36 \mathrm{~nm}$ measure at $T=300 \mathrm{~K}$. The thickness dependence of saturation magnetic moment $\left(M_{S}\right)$ and coercivity $\left(H_{C}\right)$ are shown in the panel (e) and (f), respectively.

hysteresis loops of all films measured for both in-plane and out-of-plane fields. Clearly, we observe low saturation fields and high squareness ratio for in-plane loops. While the in-plane squareness is greater than 0.7 , the out-of-plane squareness is less than 0.05 . These observations indicate that the magnetic easy axis is in the film plane. The saturation magnetization of $\mathrm{CoFeB}$ can be determined from the linear fit to the thickness dependent saturation magnet moment $\left(M_{S}\right)$. The extracted value of saturation magnetization comes out to be $1580 \mathrm{emu} / \mathrm{cc}$. Furthermore, we observe a magnetic dead layer of 0.8 $\mathrm{nm}$, which is close to the values of dead layer observed in other $\mathrm{CoFeB}$ interfaces [3]. Figure 3(f) presents the coercivity $\left(H_{C}\right)$ of easy axis loop as a function of $d$, which clearly shows the enhancement of $H_{C}$ with increasing $d$. Similar behaviour observed in various ferromagnetic alloys has been attributed to the grain size effect [26 28]. With decreasing grain size, the effective anisotropy constant reduces, which leads to increase in effective exchange range [28]. We have also observed such reduction in anisotropy with decreasing thickness (or decreasing grain size) 29]. Under such scenario, the exchangecoupling between the grains is enhanced and, thus, the $H_{C}$ reduces with decreasing thickness .

Now, we present the electrical transport study of our films [See Fig. 4(a)]. The temperature dependent fourprobe sheet resistance $R_{\square}(T)$ shows the metallic behaviour for all $\mathrm{CoFeB}$ films. The resistance decreases with lowering of the temperature up to a certain temperature, known as resistance minimum temperature $\left(T_{m i n}\right)$, and then it starts increasing down to $5 \mathrm{~K}$. The $T_{\min }$ for the $36 \mathrm{~nm}$ thick film is $26 \mathrm{~K}$ and it gradually increases with decreasing thickness [See Fig. 4(b)]. The thickness dependence of sheet conductance $G_{\square}=1 / R_{\square}$ at $T=$ $273 \mathrm{~K}$ shows a linear thickness dependence as shown in 


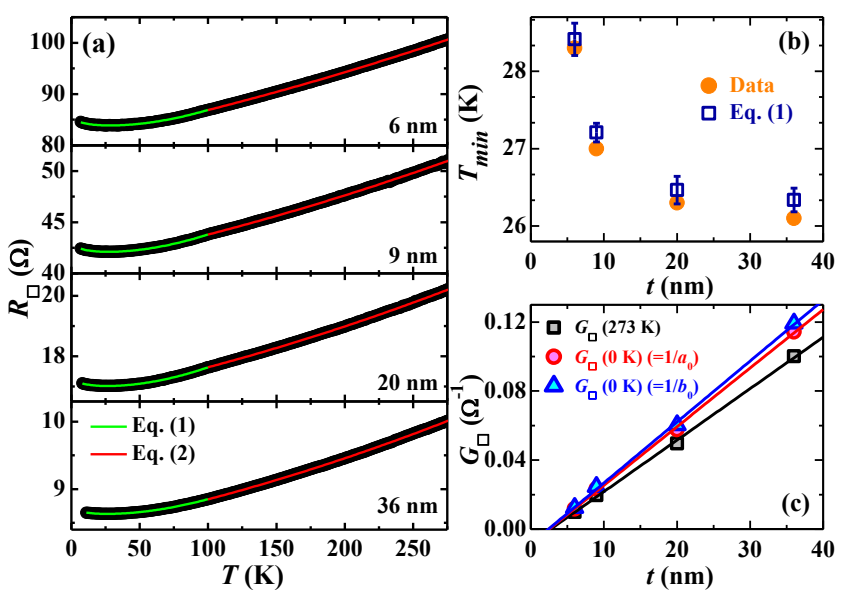

FIG. 4. (Color online) (a) Temperature dependent sheet resistance $R_{\square}(T)$ plots for $\mathrm{CoFeB}(t=6,9,20$, and $36 \mathrm{~nm})$ films. The solid lines are the fits to $R_{\square}(T)$ according to Eq. (1) and (2) with the quality of fitting given by $r^{2}>0.999$. (b) Thickness dependence of $T_{m i n}$, which is the temperature at which $\mathrm{d} R_{\square}(T) / \mathrm{d} T=0$ and $T_{\min }=\left(-\beta / 2 a_{2}\right)^{1 / 2}$ using the fitting parameters in Eq. (1). (c) Thickness dependence of $G_{\square}$ at $T=273 \mathrm{~K}$ along with $G_{\square}(0 \mathrm{~K})$ obtained from $a_{0}$ and $b_{0}$. The straight lines are the linear fits to the data.

Fig. 4(c). The slope of the linear fit yields a resistivity of $(33 \pm 2) \mu \Omega \mathrm{cm}$, which is close to previously reported values for several micron thick amorphus $\mathrm{CoFeB}$ ribbons [14]. However, one can observe a finite intercept of the linear fit on the temperature axis, which suggests the presence of an electrically less conducting dead layer. The existence of such dead layers has been reported in thin films of manganite [30] and Heusler alloy [31]. This dead layer can form on the surface and at film-substrate interface due to surface oxidation, roughness effect, and boron segregation at the interface [9, 12]. Alternatively, the presence of larger number of grain boundaries in thinner films can also result in such behavior. The thickness of the dead layer is given by the intercept on the thickness axis, which comes out as $t_{d} \simeq 2.5 \mathrm{~nm}$.

Coming back to $R_{\square}(T)$ data, we have fitted these using the following expressions. For low temperature $(5 \mathrm{~K} \leq$ $T \leq 100 \mathrm{~K}$ ) regime, the resistance is expressed as:

$$
R_{\square}(T)=a_{0}+a_{2} T^{2}+\beta \ln T,
$$

and, for high temperature (100 $\mathrm{K} \leq T \leq 273 \mathrm{~K})$ regime,

$$
R_{\square}(T)=b_{0}+b_{1} T+b_{2} T^{2},
$$

where $a_{0}, a_{2}, \beta, b_{0}, b_{1}$, and $b_{2}$ are the constants. The temperature independent terms $\left(a_{0}\right.$ and $\left.b_{0}\right)$ represent the residual sheet resistance $R_{\square}(0 \mathrm{~K})$. Using these values, the $t_{d}$ again comes out as $\simeq 2.5 \mathrm{~nm}$ [See Fig. 4(c)], which implies that the dead layer remains fixed in whole temperature range. The $T^{2}$ term in above expressions can arise due to the effects like electron-magnon scattering and electron-lattice scattering. The first scattering process is due to the coherent scattering of electrons by long-wavelength magnons in a ferromagnet, which introduces a magnetic contribution $\left(\rho_{m a g} / t\right)$ to $R_{\square}$. On the other hand, the incoherent scattering can introduce a $T^{3 / 2}$ dependent term in an amorphous ferromagnet while such process is absent in a crystalline ferromagnet [24]. Therefore, we do not observe a $T^{3 / 2}$ dependency in our fits. The latter process involves the scattering of conduction electrons from the lattice potential of a transition-metal system, which results in a structural contribution $\left(\rho_{\text {str }} / t\right)$ to $R_{\square}\left[32,33\right.$. While $\rho_{\text {mag }}$ varies as $T^{2}$ for all temperatures, $\rho_{\text {str }}$ shows a transition from $T^{2}$ to linear $T$ dependence as the temperature increases. The last term in Eq. (1) can arise due to two effects. Firstly, the logarithm dependence of resistance is a characteristics feature of Kondo scattering observed in dilute magnetic metals, quantum dots, and heavy electron systems [16]. The Kondo behavior can be explained as the interaction of conduction electrons with localized spins of magnetic impurities. Such effect can also be observed in ferromagnetic materials, where the effective field distribution for a magnetic spin has a long tail extended below zero field and, thus, some of the spins participate in spin flipping scattering process 34]. One should note that this effective field model is only valid for amorphous systems while, in crytalline ferromagnet, distinct field lines are observed in place of a field distribution 35. Also, the absence of resistance saturation (or tendency for saturation) in many reports does not provide a solid evidence of Kondo effect 14, 21, 22]. Thus, for our metallic crystalline ferromagnetic $\mathrm{CoFeB}$ thin films, the presence of Kondo effect well below its Curie temperature $\left(T_{c}\right)$ is highly unlikely. We will provide further support to our conjecture later on. The second possible explanation is the Coulomb interaction in a granular metal, where a logarithmic temperature dependent conductivity can be observed in metallic regime with dimensionless tunneling conductance $g \gg 1$ [17, 36]. To verify if this scenario is valid in our case, we have determined $g$. In the temperature range of 5 $\mathrm{K} \leq T \leq 100 \mathrm{~K}$, the maximum relative change in resistance, i.e., $\left[R_{\square}(100 \mathrm{~K})-R_{\square}\left(T_{\min }\right)\right] / R_{\square}\left(T_{\min }\right)$ is $<0.04$. For such small variation, the resistivity can be approximated to: $\rho \sim\left(\alpha / \sigma_{0}\right) \ln \left(g E_{c} / T\right)$, where $\alpha=(2 \pi g d)^{-1}$, $\sigma_{0}\left(=e^{2} g L^{2-d} / \hbar\right)$ is the conductivity of the granular metal without the Coulomb interaction, and $E_{c}$ is the charging energy of an isolated metal grain. Here, $d$ is the dimensionality of the granular array, which corresponds to the half of the number of neighboring grains to a single grain rather than to real dimensionality. Comparing this expression with the logarithmic term in Eq. (1), we have $\beta=-\left(\hbar / 2 \pi d e^{2}\right)(L / t)^{d-2} g^{-2}$. Figure 5 shows the estimated values of $g$ for $d=2$ and 3 using the fitting parameter $\beta$. Clearly, $g \gg 1$ for all the films, which indicates that the conduction is not a tunneling process through an insulator; rather a charge transfer process between the grains via metallic grain boundaries as observed in many metallic alloys [37, 38]. Moreover, the $-\beta t$ increases with decreasing thickness. This can be ex- 


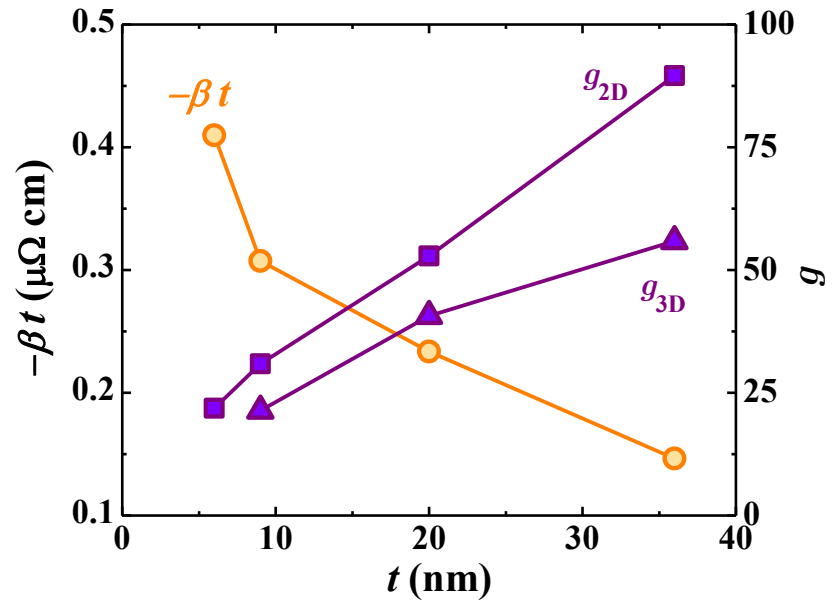

FIG. 5. (Color online) Thickness dependence of $-\beta t$ as well as $g$ for two and three dimensional granular array.
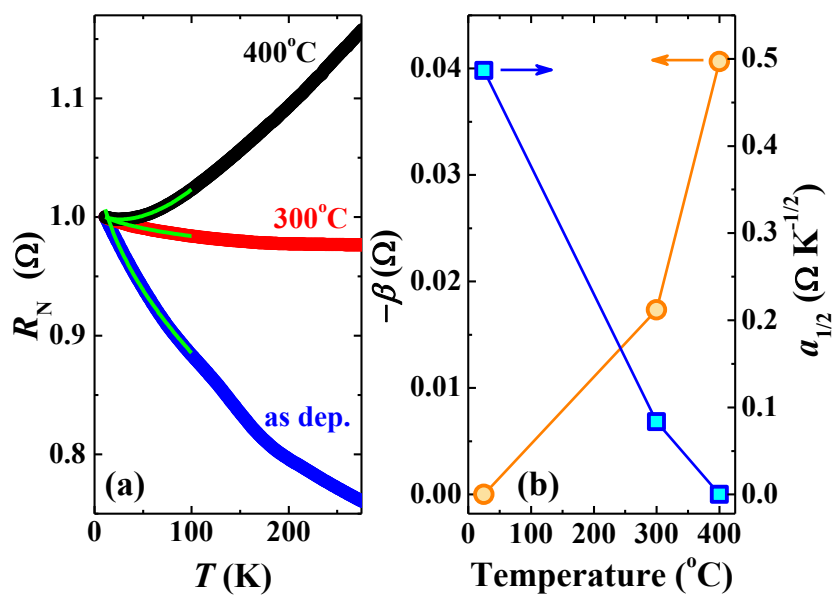

FIG. 6. (Color online) (a) The normalised sheet resistance $R_{N \square}(T)=R_{\square}(T) / R_{\square}(10 \mathrm{~K})$ of as deposited as well as annealed $40 \mathrm{~nm}$ films. The curves are fitted to the expression: $R_{\square}(T)=a_{0}+a_{1 / 2} T^{1 / 2}+a_{2} T^{2}+\beta \ln T$. The fitting parameters $a_{1 / 2}$ and $-\beta$ are plotted in the panel (b).

plained in terms of the presence of more disorders and grains in thinner films, which results in reduction of $g$ and, thereby, enhancement of $-\beta t$. All these observations indicate that the resistance minimum observed here can be due to the granular nature of the films and the enhancement of $T_{\min }$ in thinner films is due to increasing granularity and disorder. We can also determine the $T_{\min }$ using Eq. (1) as $T_{\min }=\left(-\beta / 2 a_{2}\right)^{1 / 2}$. These values match quite well with experimental values as shown in Fig. 4(b), which is an indicator of good quality of these fits.

We have analysed the normalized sheet resistance $R_{N \square}(T)$ of as deposited and annealed $36 \mathrm{~nm}$ thick films to get further insight in to the observed resistance minimum [See Fig. 6(a)]. In case of as-deposited film, we observe a negative temperature coefficient of resistance
(TCR) with a residual resistance ratio $\mathrm{RRR}=R_{N \square}(273$ $\mathrm{K}) \simeq 0.76$. Upon annealing the film at $300^{\circ} \mathrm{C}$, the RRR increases to $\simeq 0.98$ while negative TCR still persists for whole temperature range. Further annealing at $400^{\circ} \mathrm{C}$ increases the metallically of the film with $\mathrm{RRR} \simeq 1.15$ and negative TCR regime present below $26 \mathrm{~K}$. Such enhanced metallicity with thermal annealing is directly related to enhancement of crystallinity in these films [12]. While the $400^{\circ} \mathrm{C}$ annealed film is granular, the TEM image of as deposited film show a feature-less amorphous matrix with scarcely dispersed crystalline regions. So we do not expect any $\ln T$ contribution coming due to intergranular Coulomb interactions in as deposited film. On the other hand, a Kondo-type $\ln T$ dependent resistance is expected for this amorphous film, where the effective field model is valid and this effect should reduce with annealing as the film becomes gradually crystalline. In contrast, the $R_{N \square}(T)$ of this film does not fit to Eq. (1), which again points against Kondo effect in this system. We have tried to fit $R_{N \square}(T)$ by Eq. (1) with an additional $T^{1 / 2}$ term, which represents the electron-electron $(e-e)$ interactions in a three dimensional disordered system [39]. With this new expression, negative TCR in the as deposited film can be explained by only $T^{1 / 2}$ term while the $\ln T$ term vanishes [See Fig. 6(b)]. In case of $300^{\circ} \mathrm{C}$ annealed film, the $R_{N \square}(T)$ has contribution from both $\ln T$ and $T^{1 / 2}$ terms. The relative weight of these terms, i.e. $W\left(a_{1 / 2}, \beta\right)=\int a_{1 / 2} T^{1 / 2} d T / \int \beta \ln T d T$ within the limits $10 \mathrm{~K} \leq T \leq 100 \mathrm{~K}$, comes out as $\simeq 9.0$, which indicates the $e$-e interaction is still highly dominant process for $300^{\circ} \mathrm{C}$ annealed film. Olivier et al. have reported the presence of both $\ln T$ and $T^{1 / 2}$ dependent resistance in FeCrB metallic glasses, where the $T^{1 / 2}$ term is responsible for resistance minimum in some cases [23]. Thus, the presence of negative TCR in all three samples (albeit in different temperature regimes) has two different origins; i.e. e-e interactions in a disordered amorphous film and Coulomb effect in granular polycrystalline film.

In order to find out the significance of quadratic temperature dependent resistance, we have converted the coefficients $\left(a_{2}\right.$ and $\left.b_{2}\right)$ in the form of resistivities, i.e., $a_{2}^{\prime}=a_{2} t$ and $b_{2}^{\prime}=b_{2} t$. The $b_{2}^{\prime} T^{2}$ term represents the $\rho_{m a g}$ while the $a_{2}^{\prime} T^{2}$ term is a combination of $\rho_{m a g}$ and $\rho_{\text {str. }}$. Figure $7(\mathrm{a})$ shows that $b_{2}^{\prime}$ (or $\rho_{\text {mag }}$ ) remains almost constant irrespective of $t$. Such behaviour implies that all films are magnetically homogeneous, which can also be confirmed from magnetic hysteresis loop measurements shown in Fig. 3. Moreover, the disorders present in thinner films only introduce a minor correction to the magnetic $T^{2}$ term and, thus, do not significantly alter $\rho_{\text {mag }}$ [24]. The $b_{2}^{\prime}$ values are quite close to the values obtained for other ferromagnetic metals $\left(2.2-3.2 \times 10^{-11}\right.$ $\Omega \mathrm{cm} \mathrm{K}^{-2}$ for Fe and $\left.\mathrm{Co}\right)$ and alloys $\left(0.98 \times 10^{-11} \Omega\right.$ $\mathrm{cm} \mathrm{K}{ }^{-2}$ for $\left.\mathrm{Fe}_{80} \mathrm{~B}_{20}\right)$ 24, 40, 41]. The $\rho_{\text {mag }}$ is proportional to $\left(J_{s d} / D\right)^{2}$, where $J_{s d}$ is the $s$ - $d$ exchange integral and $D$ is the spin-wave stiffness constant [24]. We can estimate the values of $D$ from temperature dependent magnetization $M(T)$ data as shown in the inset of 


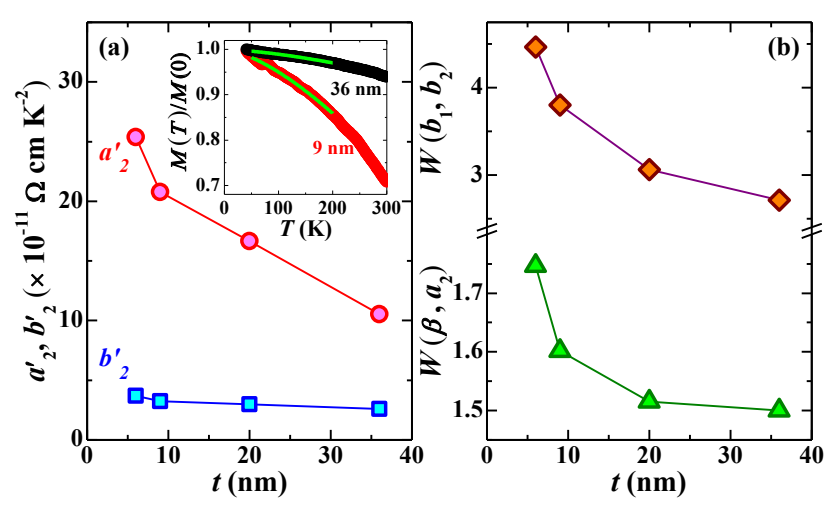

FIG. 7. (Color online) Thickness dependence of (a) $a_{2}^{\prime}, b_{2}^{\prime}$, and (b) $W\left(\beta, a_{2}\right), W\left(b_{1}, b_{2}\right)$. The inset of the panel (a) shows the $M(T) / M(0)$ curves for 6 and $36 \mathrm{~nm}$ thick films with the fits given by the relation: $M(T)=M(0)\left(1-B T^{3 / 2}\right)$.

Fig. $7(\mathrm{a})$. The $M(T)$ in the low temperature regime is given by Bloch law as follows: $M(T)=M(0)\left(1-B T^{3 / 2}\right)$, where $B=\left[\zeta(3 / 2) g_{e} \mu_{B} / M(0)\right]\left(k_{B} / 4 \pi D\right)^{3 / 2}$ [42]. Here, $\zeta(3 / 2)=2.612$ is the Riemann $\zeta$ function, $g_{e}=2$ is the gyromagnetic ratio, and $\mu_{B}$ is the Bohr magneton. Using these expressions, the $D$ comes out as 88 and $185 \mathrm{meV} \AA^{2}$ for the 9 and $36 \mathrm{~nm}$ thick films, respectively, which are comparable to the reported values for various $\mathrm{FeB}$ based alloys [42]. Since the $\rho_{\text {mag }}$ [and thereby $\left(J_{s d} / D\right)^{2}$ ] is thickness independent, $\left|J_{s d}\right|(t=9 \mathrm{~nm})<\left|J_{s d}\right|(t=36$ $\mathrm{nm})$. Here, we want to point out that the values of $J_{s d}$ are in negative. In a Kondo picture, $\beta t \propto J_{s d}^{3}$, which implies that $-\beta t(t=9 \mathrm{~nm})<-\beta t(t=36 \mathrm{~nm})$. But an opposite trend observed in our case [See Fig. 5] discards Kondo effect as the explanation of the resistance minimum. On the other hand, the low temperature $T^{2}$ coefficient $a_{2}^{\prime}$ is much larger than $b_{2}^{\prime}$ and increases with reducing $t$, which suggests an additional contribution apart from thickness independent $b_{2}^{\prime}$. This extra contribution corresponds to $\rho_{s t r}$ and it gets enhanced in thinner films due to the presence of more structural disorders and grain boundaries. Comparing these two effects, we observe a substantial magnetic contribution although $\rho_{\text {str }}$ is at least 3.5 times more dominating in comparison to $\rho_{\text {mag }}$.

Apart from the $T^{2}$ term, the $R_{\square}(T)$ expressions involve two other important terms; i.e. $\beta \ln T$ (in low $T$ regime) and $b_{1} T$ (in high $T$ regime). We have defined the relative weight of these terms with respect to corresponding $T^{2}$ terms as follows: $W\left(\beta, a_{2}\right)=\int \beta \ln T d T / \int a_{2} T^{2} d T$ and $W\left(b_{1}, b_{2}\right)=\int b_{1} T d T / \int b_{2} T^{2} d T$ with the integrations performed using the limits $5 \mathrm{~K} \leq T \leq 100 \mathrm{~K}$ and $100 \mathrm{~K} \leq T \leq 273 \mathrm{~K}$, respectively. Figure 7 (b) shows these relative weights as a function of $t$. Clearly, the Coulomb effect plays a dominating role as compared to the $T^{2}$ term at lower temperatures as $W\left(\beta, a_{2}\right)>1$. Moreover, the increase of $W\left(\beta, a_{2}\right)$ with decreasing thickness suggests that this effect becomes rather important as the grain size reduces and the grain boundary contribution increases. In high temperature regime, $\rho_{\text {str }} \sim T$ and the $W\left(b_{1}, b_{2}\right)$ basically represents the relative weight of $\rho_{s t r}$ with respect to $\rho_{\text {mag }}$. Thus, the dominating effect of linear $T$ term and its enhancement with decreasing $t$ are well expected as explained before. While the electronphonon $(e-p)$ scattering also shows a linear $T$ dependency above Bloch-Gruneisen temperature $\left(T_{B G}\right)$, it should reduce with increasing grain boundary and, thus, with decreasing thickness [43]. This behaviour is opposite to the trend seen in Fig. 7(b). Moreover, the $e-p$ scattering in low $T$ regime $\left(T<<T_{B G}\right)$ results in a $T^{5}$ dependent resistance term. We have not observed any improvement to the fits by additional introduction of this term in Eq. (1). Therefore, one can safely assume that the electronphonon scattering does not play an important role in the electronic conduction.

In conclusion, we have investigated pulsed laser deposited polycrystalline $\mathrm{CoFeB}$ films of varying thickness. The analysis of transport measurements of $\mathrm{CoFeB}$ thin films demonstrates the effect of granularity and disorder on the structural, magnetic, and Coulomb interaction related scattering processes. The resistance minimum is related to the grains (and the grain boundaries) present in the film. While the magnetic contribution to resistance remains independent of the film thickness, the structural contribution and inter-granular Coulomb effect increases with decreasing thickness. In amorphous films, the electrical conduction is mainly dominated by the electron interaction effects in disordered systems. Our comprehensive study of electronic transport in $\mathrm{CoFeB}$ films shows that the electrical conduction in thinner films will be affected by granularity (or Coulomb charging effects). Therefore, better performance of spintronics devices can be achieved either by reducing device size to the order of grain size or by developing a better fabrication technique for thin films with large grains.

We thank R. C. Budhani for his valuable comments, K. K. Maurya for XRD measurements, and V. Toutam for AFM measurements. We acknowledge Council of Scientific and Industrial Research (CSIR) \& Department of Science and Technology (DST) for financial support.

* pkrout@mail.nplindia.org

[1] Ikeda S et al 2006 Tunnel magnetoresistance of $604 \%$ at $300 \mathrm{~K}$ by suppression of Ta diffusion in $\mathrm{CoFeB} / \mathrm{MgO} / \mathrm{CoFeB}$ pseudo-spin-valves annealed at high temperature Appl. Phy. Lett. 93082508

[2] Wang W G et al 2010 Understanding tunneling magnetoresistance during thermal annealing in $\mathrm{MgO}$-based junctions with CoFeB electrodes Phys. Rev. B 81144406

[3] Ikeda $\mathrm{S}$ et al 2010 A perpendicular-anisotropy CoFeBMgO magnetic tunnel junction Nature. Mater. 9721

[4] You C Y et al 2008 Exchange bias of spin valve structure with a top-pinned $\mathrm{Co}_{40} \mathrm{Fe}_{40} \mathrm{~B}_{20} / \mathrm{IrMn}$ Appl. Phys. Lett. 93012501

[5] Tulapurkar A A et al 2005 Spin-torque diode effect in magnetic tunnel junctions Nature 438339 
[6] Liu Luqiao et al 2012 Spin-torque switching with the giant spin Hall effect of tantalum Science 336 555-558

[7] Fujimori H et al 1994 High electrical resistivity and permeability of soft magnetic granular alloys IEEE Trans. Magn. 304779

[8] Johnsson $\mathrm{P}$ et al 2003 Transport anisotropy in heteroamorphous (CoFeB)- $\mathrm{SiO}_{2}$ thin films J. Appl. Phys. 93 8101

[9] Jen S U et al 2006 Magnetic and electrical properties of amorphous CoFeB films J. Appl. Phys. 99053701

[10] Deng Lianwen et al 2007 Percolation and microwave characteristics of $\mathrm{CoFeB}-\mathrm{SiO}_{2}$ nano-granular films $J$. Magn. Magn. Mater. 309285

[11] Huang S X et al 2008 Spin polarization of amorphous $\mathrm{CoFeB}$ determined by point-contact Andreev reflection Appl. Phys. Lett. 92242509

[12] Venkat Swamy G et al 2013 Effect of thermal annealing on Boron diffusion, micro-structural, electrical and magnetic properties of laser ablated $\mathrm{CoFeB}$ thin films $A I P$ Adv. 3072129

[13] Ruffer Daniel et al 2014 Anisotropic magnetoresistance of individual $\mathrm{CoFeB}$ and $\mathrm{Ni}$ nanotubes with values of up to $1.4 \%$ at room temperature APL Mater. 2076112

[14] Kettler W et al 1982 Electrical resistivity and thermopower of amorphous $\mathrm{Fe}_{x} \mathrm{Co}_{80-x} \mathrm{~B}_{20}$ alloys J. Appl. Phys. 538248

[15] Efros A L et al 1975 Coulomb gap and low temperature conductivity of disordered systems J. Phys. C: Solid State Phys. 8 L49

[16] Kondo J et al 1964 Resistance minimum in dilute magnetic alloys Prog. Theor. Phys. 3237

[17] Efetov K B et al 2003 Coulomb effects in granular materials at not very low temperatures Phys. Rev. B 67 174205

[18] Cochrane R W et al 1975 Structural manifestations in amorphous alloys: Resistance minima Phys. Rev. Lett. 35676

[19] Hasegawa R et al 1970 s-d exchange interaction in amorphous Cr-Pd-Si and Mn-Pd-Si Alloys Phys. Rev. B 2 1631

[20] Hasegawa Ryusuke et al 1971 Kondo effect in amorphous Fe-Pd-Si and Co-Pd-Si alloys Phys. Rev. B 3214

[21] Fernndez Barqun L et al 1990 Electrical resistivity between 10 and $1000 \mathrm{~K}$ of ferromagnetic $\mathrm{Co}_{75} \mathrm{Si}_{25-x} \mathrm{~B}_{x}$ and $\mathrm{Co}_{100-x}\left(\mathrm{Si}_{0.6} \mathrm{~B}_{0.4}\right)_{x}$ amorphous ribbons J. Appl. Phys. 684610

[22] Shen Bao-gen et al 1997 Temperature dependence of the electrical resistivity of amorphous $\left(\mathrm{Fe}_{1-x} \mathrm{Co}_{x}\right)_{78} \mathrm{Si}_{9.5} \mathrm{~B}_{12.5}$ alloys J. Appl. Phys 814661

[23] Olivier $\mathrm{M}$ et al 1987 Disorder and magnetic effects on the electron-transport properties of iron-chromiumboron metallic glasses Phys. Rev. B 35333

[24] Kaul S N et al 1986 Evidence for a magnetic contribution to the electrical resistivity in amorphous $\mathrm{Fe}_{80} \mathrm{~B}_{20-x} \mathrm{C}_{x}$ a11oys Phys. Rev. B 334987

[25] Kaul S N et al 1987 Electrical resistivity of amorphous $\mathrm{Fe}_{82} \mathrm{~B}_{18-x} \mathrm{Ge}_{x}$ alloys: Coherent electron-magnon scattering contribution Phys. Rev. B 357153

[26] Sun W S et al 2006 Thermal stability and magnetic properties of Co-Fe-Hf-Ti-Mo-B bulk metallic glass Intermetallics 1410661068

[27] Chen Yuan-Tsung et al 2010 Effect of grain size on magnetic and nanomechanical properties of $\mathrm{Co}_{60} \mathrm{Fe}_{20} \mathrm{~B}_{20}$ thin films J. Alloys and Compounds 498113117

[28] Yao Dongsheng et al 2010 Grain size dependence of coercivity in magnetic metal-insulator nanogranular films with uniaxial magnetic anisotropy J. Appl. Phys. 107 073902

[29] Venkat Swamy G et al 2015 Origin of in-plane and outof-plane magnetic anisotropies in as-deposited and annealed CoFeB ferromagnetic thin films J. Appl. Phys. $11717 \mathrm{~A} 312$

[30] Sun J Z et al 1999 Thickness-dependent magnetotransport in ultrathin manganite films Appl. Phys. Lett. 74 3017

[31] Rout P K et al 2014 Two-dimensional electron-gas-like charge transport at the interface between a magnetic Heusler alloy and $\mathrm{SrTiO}_{3}$ Phys. Rev. B 89 020401(R)

[32] Cote P J et al 1977 Resistivity in amorphous and disordered crystalline alloys Phys. Rev. Lett. 39102

[33] Cote P J et al 1978 Origin of saturation effects in electron transport Phys. Rev. Lett. 401586

[34] Grest G et al 1979 Effective-field distributions and resistivity minima in amorphous ferromagnets Phys. Rev. B 193571

[35] Chien C L et al 1979 Magnetic properties of amorphous $\mathrm{Fe}_{x} \mathrm{~B}_{100-x}(72<\mathrm{x}<86)$ and crystalline $\mathrm{Fe}_{3} \mathrm{~B}$ Phys. Rev. $B$ 20283

[36] Beloborodov I S et al 2007 Granular electronic systems Rev. Mod. Phys. 79469

[37] Q. Xiao John et al 1992 Giant magnetoresistance in nonmultilayer magnetic systems Phys. Rev. Lett. 683749

[38] Q. Xiao John et al 1992 Giant magnetoresistance in the granular Co-Ag system Phys. Rev. B 46 9266(R)

[39] A. Lee Patrick et al 1985 Disordered electronic systems Rev. Mod. Phys. 57287

[40] Raquet B et al 2002 Electron-magnon scattering and magnetic resistivity in 3d ferromagnets Phys. Rev. B 66 024433

[41] Goodings D A et al 1963 Electrical resistivity of ferromagnetic metals at low temperatures Phys. Rev. 132542

[42] Fernandez-Baca J A et al 1987 Long-wavelength spinwave energies and linewidths of the amorphous Invar alloy $\mathrm{Fe}_{100-x} \mathrm{~B}_{x}$ Phys. Rev. B 368497

[43] Boekelheide Z et al 2009 Resonant impurity scattering and electron-phonon scattering in the electrical resistivity of Cr thin films Phys. Rev. B 80134426 